Z. klin. Chem. u. klin. Biochem.

7. Jg., S. 269-274, Mai 1969

\title{
Analogrechneranalyse über den Einfluß des Insulins auf den Galaktosestoffwechsel des Menschen
}

\author{
Von K. Rommel und K. GRIMmeL ${ }^{1}$ )
}

Aus der Sektion für Klinische Laboratoriumsdiagnostik und der Abteilung für Endokrinologie und Stoffivechsel (Prof. Dr. E. F. Pfeiffer) des Zentrums für Innere Mediqin der Universität Ulm (Medizinisch-Naturwissenschaftliche Hochscbule)

(Eingegangen am 27. Dezember 1968)

Die Erniedrigung des Galaktoseblutspiegels nach oraler Galaktosebelastung durch Insulin ist hervorgerufen durch Erhöhung des Transfers der Galaktose aus der Blutbahn und Hemmung der Galaktoseresorption im Darm. Für diese Untersuchungen wurde ein Analogrechnermodell entwickelt, das es gestattet, die Zeitkonstanten für Galaktose in den Kompartimenten Magen, Darm, Leber und Blut aus dem Blutspiegel zu analysieren. Die Computeranalyse des Blutspiegels oral gegebener Stoffe stellt ein prinzipielles Modell dar, das der Verdauungs-, Resorptions- und Stoffwechselforschung, vor allem in der klinischen Medizin, neue Möglichkeiten eröffnet.

\section{Analogue computer analysis of the effect of insulin on the metabolism of galactose in bumans}

The depression of the concentration of blood galactose after oral loading with galactose and intravenous injection of insulin is elicited by an increase in the transfer of galactose from the bloodstream and the restriction of galactose absorption from the small intestine. An analog computer model was developed for these investigations which permitted analysis from the blood level of the time spent by galactose in the stomach, small intestine, liver and blood. Computer analysis of the blood level of orally administered substances represents a basic model that evokes new possibilities for research in digestive absorptive and metabolic processes in clinical medicine.

\section{Einfluß des Insulins auf den Galaktosestoffwechsel}

Nach Insulingabe findet man sowohl nach oraler Verabfolgung $(1,2)$ als auch nach intravenöser Galaktosegabe $(1,3)$ eine abgeflachte Galaktoseblutspiegelkurve und eine verminderte Galaktoseausscheidung im Harn. Ursache der abgeflachten Blutspiegelkurve kann eine verminderte Resorption, gesteigerte Elimination (Abbau oder Ausscheidung im Harn) oder eine Vergrößerung des Verteilungsvolumens sein. Der Einfluß von Insulin auf die Galaktoseresorption des Menschen wurde unseres Wissens bisher nicht untersucht. BEYREISS, MÜlLER und STRACK (4) fanden am intakten Kaninchen eine Hemmung der Galaktoseresorption um $30 \%$. v. UexKüLL (5) fand, daß bei gleichzeitiger Galaktose- und Insulingabe mehr Glykogen in der Kaninchenleber gebildet wird als bei Galaktosegabe allein. Untersuchungen an eviscerierten und nephrektomierten Kaninchen, Hunden und Ratten (6-9) zeigten, daß durch Insulin der Galaktoseaustritt aus dem Blut beschleunigt und das Galaktose-Verteilungsvolumen vergrößert wird, und zwar von $40-45 \%$ auf $70 \%$ des Körpergewichtes. Damit verteilt sich die Galaktose unter der Insulinwirkung auf das gesamte Körperwasser; das Insulin muß also einen Transfer der Galaktose ins Zellinnere bewirken. Denselben Effekt zeigt auch die Glucose, nicht dagegen die Fructose. Insulin führt aber nach diesen Autoren nicht zu einer Galaktoseutilisation; der Gesamt-Galaktosegehalt bleibt nämlich gleich. WICK und DRURY (10) fanden dagegen an eviscerierten Kaninchen auch einen Stoffwechseleffekt des Insulins; die Galaktoseoxydation wurde auf das Doppelte gesteigert.

1) Mit Unterstützung der Deutschen Forschungsgemeinschaft Bad Godesberg.
Da Galaktose beim Menschen über Umwandlung in Glucose zu einer Insulinsekretion führt (11) soll untersucht werden, welchen Einfluß parenteral zugeführtes Insulin auf den Galaktoseumsatz hat.

Bei der Bearbeitung dieses Problems wurde von dem Gedanken ausgegangen, mit Hilfe biokinetischer Verfahren aus dem Blutspiegel Auskunft über den Komplex miteinander in Beziehung stehender dynamischer $\mathrm{Ab}$ läufe zu erhalten. Hierbei ist es notwendig festzustellen, $\mathrm{da} \beta$ der Blutspiegel eine Folge der Einflüsse ist, die die betreffende Substanz nach oraler Gabe bis zu ihrem Erscheinen im Blutkreislauf erfährt. Daraus folgt, daß aus dem Blutspiegel nach Anfertigung eines den Vorgang nachahmenden Modells das Schicksal, das die Substanz auf dem Wege zum Blutkreislauf erfahren hat, analysiert werden kann.

Die Analyse aus dem Blutspiegelverlauf vorzunehmen ist nicht nur sympatisch, sondern auch sinnvoll, weil die Untersuchungstechnik nur eine geringe Belästigung des Probanden bedeutet, der Einfluß von Manipulationen mit Sonden entfällt und hier auch Organfunktionen meßbar werden, die einer direkten Messung nur schwer zugänglich und mit Risiken belastet sind (z. B. Lebervenenkatheterismus).

\section{Analogrechnermodell des Galaktosestoffwechsels nach oraler Galaktosegabe}

Das der mathematischen Analyse zugrunde liegende Modell gründet sich auf den folgenden physiologischbiochemischen Ablauf des Galaktosestoffwechsels. Oral aufgenommene Galaktose wird im extrakorporalen Raum von Magen und Darm transportiert, von der Mukosazelle resorbiert, durch die Pfortader zur Leber gebracht, dort zum Teil verstoffwechselt, zum Teil ins 
periphere Blut abgegeben. Der Blutspiegel ist eine Funktion dieser verschiedenen Vorgänge. Für die mathematische Formulierung unterteilen wir den physiologischen Ablauf von der oralen Aufnahme der Galaktose bis zu ihrem Erscheinen im Blut in vier gesondert zu betrachtende Teilvorgänge. Jeder Teilvorgang läuft in einem gesonderten Raum mit seiner eigenen Kinetik (Compartment) ab. Die vier Compartimente des Modells sind Magen, Darm, Leber und Blut, die als Einwegsystem hintereinandergeschaltet sind. Diese Teilvorgänge sollen im folgenden näher betrachtet werden:
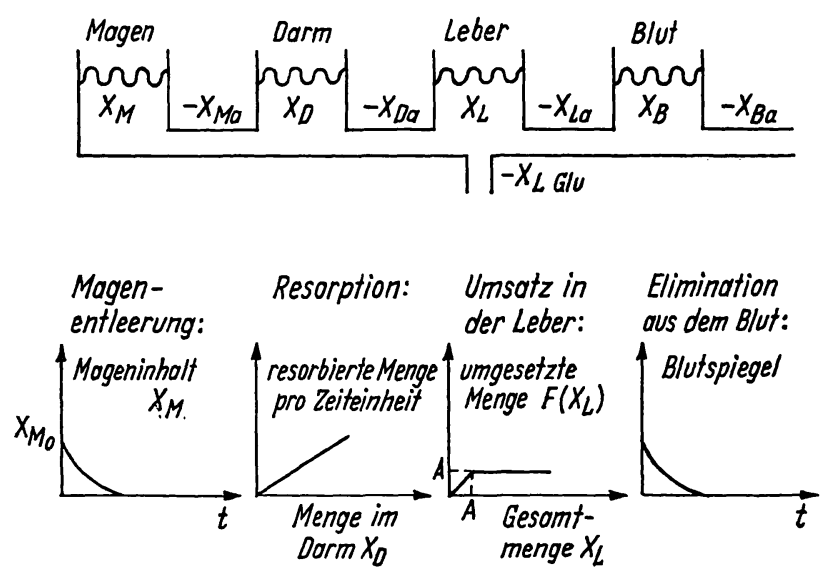

Abb. 1

Compartment-Modell des Galaktoseumsatzes

1. Der Mageninhalt entleert sich exponentiell in den Darm (12). Es wird eine vollständige Abgabe der oral gegebenen Dosis an den Darm angenommen (13).

Die mathematische Formulierung lautet dann:

$$
\begin{gathered}
X_{M I}=X_{M F} \cdot e-K_{m} \cdot t \\
\frac{d x_{M I}}{d t}=-k_{M} \cdot X_{m}=-\frac{d x_{M a}}{d t} .
\end{gathered}
$$

Dabei bedeuten:

$$
\begin{aligned}
\mathrm{X}_{\mathrm{M}}= & \text { die zur Zeit } \mathrm{t} \text { im Magen befindliche Galaktose- } \\
& \text { menge, } \\
\mathrm{X}_{\mathrm{Ml}}= & \text { die in den Magen applizierte Galaktose- } \\
& \text { gesamtmenge }(=40 \mathrm{~g}), \\
\mathrm{X}_{\mathrm{Ma}}= & \text { die zur Zeit } \mathrm{t} \text { in den Darm abgegebene } \\
& \text { Galaktosemenge und } \\
\mathrm{K}_{\mathrm{MI}}= & \text { die Eliminationskonstante des Magens für } \\
& \text { Galaktose. }
\end{aligned}
$$

2. Die pro Zeiteinheit im Darm resorbierte Galaktosemenge ist direkt proportional der zur Resorption angebotenen Menge Galaktose im Darm (14). Die Resorption der Galaktose ist vollständig. Es erfolgt kein Verlust mit dem Stuhl (14-16).

Mathematisch läßt sich dies so formulieren:

$$
\begin{aligned}
& \frac{d x_{D}}{d t}=-K_{D} \cdot x_{D}+\frac{d x_{M a}}{d t}=-K_{D} \cdot x_{D}+K_{M} \cdot x_{M} \\
& \frac{d x_{D a}}{d t}=K_{D} \cdot x_{D} .
\end{aligned}
$$

Dabei bedeuten neben den schon definierten Symbolen:

$\mathbf{x}_{\mathbf{D}}=$ die zur Zeit $\mathrm{t}$ im Darm befindliche Galaktosemenge,

$\mathrm{x}_{\mathrm{Da}}=$ die zur Zeit $\mathrm{t}$ vom Darm in die Vena portae bzw. die Leber abgégebene Galaktosemenge und

$\mathrm{k}_{\mathrm{D}}=$ die Eliminationskonstante des Darmes für Galaktose.

3. Die gesamte applizierte Galaktosemenge gelangt über die Pfortader in die Leber. Hier erfolgt die fermentative Umwandlung der Galaktose in Glucose, die einer Suibstratsättigungskinetik gehorcht; das heißt, jenseits einer bestimmten in der Leber befindlichen Galaktosemenge $A$ ist die pro Zeiteinheit in Glucose umgewandelte Galaktosemenge konstant (17).

Die mathematische Formulierung dieser physiologischen Vorgänge lautet:

$$
\begin{gathered}
\frac{d x_{I}}{d t}=-k_{L} \cdot x_{L}-\frac{d x_{L G l u}}{d t}+\frac{d x_{D_{2}}}{d t}=-k_{L} \cdot x_{I} \\
-F\left(x_{L}\right)+k_{D} \cdot x_{D} .
\end{gathered}
$$

Dabei bedeuten - neben den oben bereits definierten Symbolen:

$$
\begin{aligned}
\mathrm{x}_{\mathrm{L}}= & \text { die zur Zeit } \mathrm{t} \text { in der Leber befindliche Galak- } \\
& \text { tosemenge, } \\
\mathrm{x}_{\mathrm{LGlu}}= & \text { die zur Zeit } \mathrm{t} \text { in Glucose umgewandelte } \\
& \text { Galaktosemenge, } \\
\mathrm{k}_{\mathrm{L}}= & \text { die Eliminationskonstante der Leber für die } \\
& \text { Galaktosegabe ins Blut und } \\
\mathrm{F}\left(\mathrm{x}_{\mathrm{L}}\right)= & \text { die mathematische Formulierung der Leber- } \\
& \text { schwelle bzw. der Sättigungskinetik: } \\
\mathrm{F}\left(\mathrm{x}_{\mathrm{L}}\right)= & \left\{\begin{array}{l}
0 \text { für } \mathrm{x}_{\mathrm{L}}=0 \\
\beta \cdot \mathrm{x}_{\mathrm{L}} \text { für } 0<\mathrm{x}_{\mathrm{L}}<\mathrm{A} \\
\beta \cdot \mathrm{A} \text { für } \mathrm{x}_{\mathrm{L}} \geqq \mathrm{A}
\end{array}\right.
\end{aligned}
$$

wobei A die in der Leber befindliche Galaktosemenge bedeutet, bei der die maximale Umsatzkapazität der Leber für Galaktose erreicht wird und $\beta$. $x_{\mathrm{I}}$ im Bereich $x_{\mathbf{L}}<\mathrm{A}$ die pro Zeiteinheit in Glucose umgewandelte Galaktosemenge angibt, wobei $\beta$ die Umsatzkonstante ist.

4. Die exponentielle Elimination der Blutgalaktose wird überlagert durch die gleichzeitige Galaktosezufuhr über die Leber. Mathematisch formuliert, ergibt sich daraus die Gleichung:

$$
\frac{d x_{B}}{d t}=-k_{B} \cdot x_{B}+k_{I} \cdot x_{I} .
$$

Dabei bedeuten - neben dẹn schon bekannten Lebersymbolen: 
$\mathbf{x}_{\mathrm{B}}=$ die zur Zeit $\mathrm{t}$ im Blut befindliche Galaktosemenge und

$\mathrm{k}_{\mathrm{B}}=$ die Eliminationskonstante der Galaktose für das Blut.

Man erhält also nach dieser Modell-Vorstellung die jeweils zur Zeit $t$ im Blut befindliche Galaktosemenge in Gramm. Die experimentell gemessene Blutspiegelkurve in $\mathrm{mg} / 100 \mathrm{ml} \mathrm{mu}$ deshalb zum Vergleich ebenfalls auf die Galaktosemenge in Gramm umgerechnet werden, wobei wir ein Blutvolumen von $1 / 12$ des Körpergewichtes angenommen haben. Bei einem durchschnittlichen Körpergewicht von $70 \mathrm{~kg}$ wäre demnach mit einem Blutvolumen von rund $6 / \mathrm{zu}$ rechnen. Der maximale Blutspiegel von $50 \mathrm{mg}$ Galaktose pro $100 \mathrm{ml}$ Vollblut entspräche damit einer Galaktosemenge von $3 \mathrm{~g}$. Die am Analogrechner eingestellte experimentell gewonnene Meßkurve hat dann ein Maximum von 0,75 bezogen auf $4 \mathrm{~g}=1$. Um die oben aufgestellten Differentialgleichungen in den Analogrechner geben $z u$ können, müssen sie normiert, d. h. rechentechnisch verwertbar und dimensionslos gemacht werden. Dazu gelten folgende, neue Beziehungen:

$$
\mathrm{X}_{\overline{\mathbf{M}}, \mathrm{D}, \mathrm{I}, \mathbf{B}}=\frac{\mathrm{X}_{\mathrm{M}, \mathrm{D}, \mathrm{I}, \mathrm{B}}}{\mathrm{X}_{\mathrm{Ma}}}
$$

In unserem Falle ist $\mathrm{x}_{\mathrm{Ma}}=40 \mathrm{~g}$.

$$
\tau=\frac{\mathrm{t}}{120 \mathrm{Min} .} \text {. }
$$

Es ist $z w e c k m a ̈ ß i g$, die Echtzeit $t$ auf die bei dieser Untersuchung gewählte gesamte Versuchszeit von 120 Min. zu normieren. Die Zeitvariable $\tau$ hängt dann mit dem Zeitablauf $t^{*}$ im Analogrechner durch die Beziehung $=\tau=\frac{\mathrm{t}^{*}}{1 \text { Sek. }}$ zusammen, das heißt, das Zeitintervall von 120 Min. läuft in 1 Sek. ab.

Das normierte Gleichungssystem lautet nun:

1. $\frac{\mathrm{dX} \overline{\mathrm{M}}}{\mathrm{d} \tau}=-\mathrm{k}_{\mathrm{M}}^{*} \cdot \mathrm{X}_{\overline{\mathrm{M}}}$

$$
\mathrm{k}_{\mathrm{M}}^{*}=120 \mathrm{Min} \cdot \mathrm{k}_{\mathrm{M}}
$$

2. $\frac{\mathrm{dX}_{\overline{\mathrm{D}}}}{\mathrm{d} \tau}=\mathrm{k}_{\mathrm{D}}^{*} \cdot \mathrm{X}_{\overline{\mathrm{D}}}+\mathrm{k}_{\mathrm{M}}^{*} \cdot \mathrm{X}_{\overline{\mathrm{M}}}$

$$
k_{\mathbf{D}}^{*}=120 \mathrm{Min} \cdot \cdot k_{\mathbf{D}}
$$

3. $\frac{\mathrm{dX}_{\mathrm{L}}}{\mathrm{d} \tau}=-\mathrm{k}^{*} \cdot \mathrm{X}_{\overline{\mathrm{L}}}-\mathrm{F}^{*}\left(\mathrm{X}_{\overline{\mathrm{L}}}^{-}\right)+\mathrm{k}_{\mathrm{D}}^{*} \cdot \mathrm{X}_{\overline{\mathrm{D}}}$

$$
\begin{aligned}
\mathrm{F}^{*}\left(\mathrm{X}_{\overline{\mathrm{L}}}\right) & =\frac{120 \text { Min. }}{\mathrm{X}_{\mathrm{Mo}}} \cdot \mathrm{F}\left(\mathrm{X}_{\mathrm{Mo}} \cdot \mathrm{X}_{\overline{\mathrm{L}}}\right) \\
\mathrm{k}_{\mathrm{L}}^{*} & =120 \mathrm{Min.} \cdot \mathrm{k}_{\mathrm{L}} \\
\mathrm{F}^{*}\left(\mathrm{X}_{\mathrm{L}}\right) & =\left\{\begin{array}{l}
\text { o für } \mathrm{X}_{\overline{\mathrm{L}}}<0 \\
\beta^{*} \cdot \mathrm{X}_{\overline{\mathrm{L}}} \text { für } 0 \leqq \mathrm{X}_{\overline{\mathrm{L}}} \leqq \mathrm{A}^{*} \\
\beta^{*} \cdot \mathrm{A}^{*} \text { für } \mathrm{X} \overline{\mathrm{L}}>\mathrm{A}^{*}
\end{array}\right.
\end{aligned}
$$

\section{Material und Methode}

Die Untersuchungen wurden an 12 gesunden - insbesondere lebergesunden - männlichen Personen im Alter von 20 bis 40 Jahren durchgeführt.

Als lebergesund wurden Personen befunden, die anamnestisch keine Lebererkrankung durchgemacht hatten und bei denen die Leberfunktionsproben (Elektrophorese, die Transaminasen, SGOT und SGPT, alkalische Phosphatase und Bromthaleintest) im Normbereich lagen. Sämtliche Probanden hatten einen normalen Glucoseassimilationskoeffizienten. Alle Belastungen wurden an nüchternen Personen (letzte Nahrungsaufnahme mindestens $12 \mathrm{Stdn}$. vor Versuchsbeginn) morgens unter Grundumsatzbedingungen durchgeführt. Jeder Proband wurde zwei Belastungen unterzogen:

1. Mit Galaktose,

2. Mit Galaktose + Insulin (0,1 E Altinsulin/kg Körpergewicht i. v.).

Zwischen den einzelnen Belastungen wurde ein Intervall von mindestens 4 Tagen eingehalten.

Im ersten Versuch wurde $40 \mathrm{~g}$ Galaktose in $200 \mathrm{~m} /$ Wasser gelöst oral zugeführt, nachdem Kapillarblut aus der Fingerbeere für die Nüchternwerte entnommen war. Bis 90 Min. nach Zufuhr der Galaktose exfolgten die Blutentnahmen zur Bestimmung der Galaktose und Glucose in 15 minütigem Abstand, im Verlauf weiterer 90 Min. wurde das Blut in Abständen von 30 Min. entnommen. Nach Ablauf der 180 Min. wurde die Galaktoseausscheidung im Urin mit Hilfe der klassischen Polarisationsmethode bestimmt.

Im zweiten Versuch wurde nach oraler Gabe von $40 \mathrm{~g}$ Galaktose $0,1 \mathrm{E}$ Altinsulin/kg Körpergewicht in 1 Min. intravenös rasch injiziert. Die Blutentnahmen erfolgten wie oben beschrieben. Die Galaktose wurde im Blut nach der Methode von ROMMEL, BERNT, Schmitz und Grimmel (18) enzymatisch bestimmt.

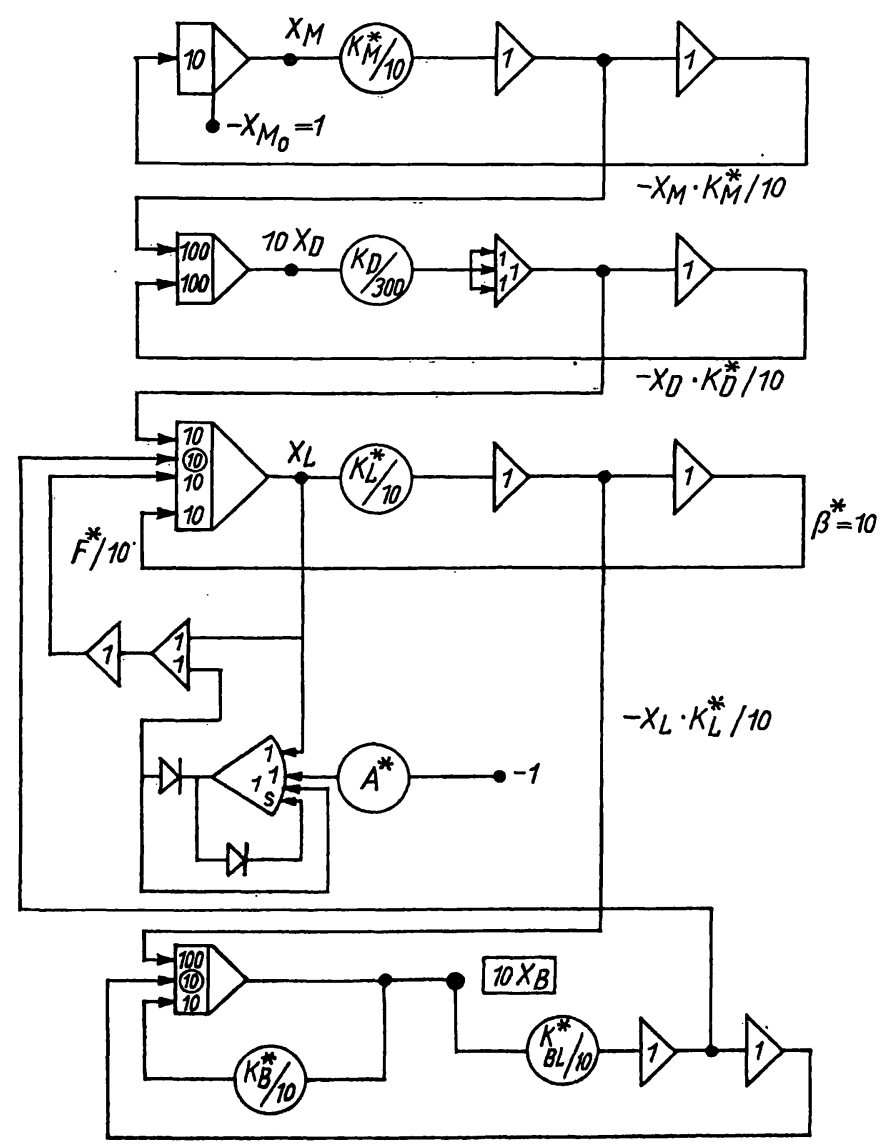

Abb. 2

Rechenschaltung des Analogrechnermodells 
Untersucbungen des Insulineinflusses auf den Galaktoseumsatz nacb oraler Galaktosegabe mit dem Analogrecbner

Die Blutgalaktosemittelwertskurven vor und nach Insulingabe wurden der Analogrechneranalyse unterzogen ${ }^{2}$ ). Dabei erwarteten wir, daß die Analyse der Galaktosekinetik in den vier Kompartimenten Magen, Darm, Leber und Blut eine Aussage über die Art des Insulineinflusses gestattet. Diese Untersuchungen wurden mit dem Analogrechner "Telefunken RA 800" durchgeführt, der mit einem Schreiber gekoppelt war (Rechenschaltung Abb. 2).

Die Mittelwertskurve des Galaktoseblutspiegels ohne gleichzeitige Insulingabe wurde in den Rechner eingegeben und nach dem geschilderten Modell simuliert, das heißt, die Modellkurve wurde mit der eingegebenen experimentellen Kurve am Oszillographen zur bestmöglichen Deckung gebracht durch Änderung der Potentiometereinstellungen. Die Potentiometerstellungen wurden dann abgelesen und die beiden Kurven mit dem Schreiber auf Papier aufgezeichnet. In gleicher Weise wurde mit der Mittelwertskurve des Galaktoseblutspiegels nach Insulingabe verfahten. Die Kurvenabweichung der eingegebenen Kurve mit der simulierten Kurve betrug maximal $\pm 4 \%$.

\section{Ergebnisse}

Der Verlaưf der eingegebenen und simulierten Kurven und die Mengenverhältnisse der Galaktose in den einzelnen Kompartimenten vor und nach Insulingabe sind aus den Abbildungen 3 und 4 ersichtlich. In Tabelle 1 sind die kinetischen Größen bei oraler Galaktosebelastung ohne und mit gleichzeitiger Insulingabe dargestellt. Die Simulation der Kurve vor Insulingabe unterscheidet sich von der Simulation der Kurve nach Insulingabe nur durch Änderung der Potentiometerstellung „Darm“ $\left(\mathrm{K}_{\mathrm{D}}\right)$ und „Blut" $\left(\mathrm{K}_{\mathrm{B}}\right)$. Daraus folgt, daß die Kinetik der Kompartimente Darm und Blut durch Insulin beeinflußt wird, die Kinetik der Kompartimente Magen und Leber unbeeinflußt bleibt.

Während $K_{D}$ ohne Insulin 1,6750 und die entsprechende Zeitkonstante (ZK) 0,60 betragen, liegen diese Werte unter Insulingabe bei 0,1250 und 8,00. Daraus folgt, daß es unter Insulin zu einer stark verzögerten Galaktoseresorption im Darm kommt. Das bedeutet einen vermehrten Galaktosegehalt des Darmes, ein niedrigeres und verspätetes Galaktosemaximum in der Leber und schließlich einen niedrigeren Galaktoseblutspiegel. Auch die $\mathrm{K}_{\mathbf{B}}$-Werte unterscheiden sich erheblich. Ohne Insulin beträgt $\mathrm{K}_{B} 0,0675 \mathrm{bzw}$. ZK 14,8 nach Insulin $0,1100 \mathrm{bzw}$. 9,1. Unter Insulin kommt es also zu einer schnelleren Galaktoseelimination aus dem Blut. Die am Analogrechner ermittelte Zeitkonstante (ZK) des Magens vor und nach Insulingabe beträgt 31,7. Das bedeutet, daß die Magenentleerung einer exponentiellen

2) Herrn Dipl.-Ing. Kley, Forschungsinstitut Telefunken, Ulm, danken wir für seine freundliche Unterstützung.

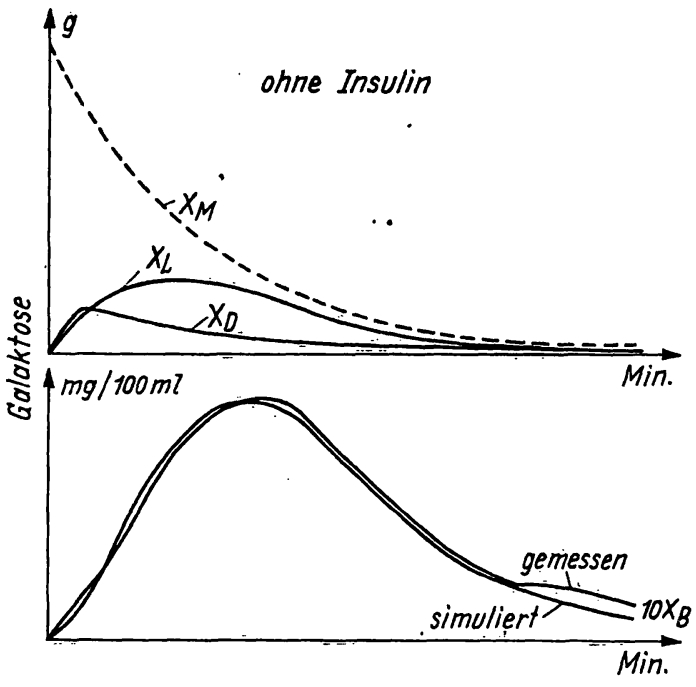

Abb. 3

Simulation des Galaktoseumsatzes (unten) und Compartmentanalysen (oben) vor Insulingabe

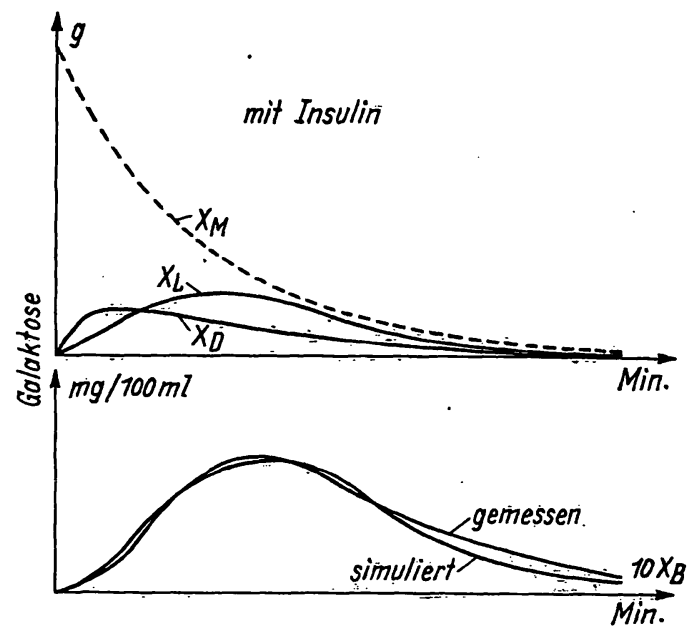

Abb. 4

Simulation des Galaktoseumsatzes (unten) und Compartmentanalysen (oben) nach Insulingabe

Funktion gehơrcht (12), daß in 31,7 Min. der Mageninhalt auf $\frac{1}{\mathrm{e}} \approx \frac{1}{3}$ des Ausgangsvolumens abgenommen hat.

Abbildung 5 zeigt, daß unserer Modell auch für die intraduodenale Applikation der Galaktose gilt. Hierbei wird sinngemä $\beta$ das Kompartiment Magen weggelassen und davon ausgegangen, daß sich zur Zeit $t_{0}$ die Galaktosemenge $\mathrm{X}_{\mathrm{MO}}$ schon im Darm befindet.

Zusammenfassend ergibt die Analogrechneranalyse des Galaktoseumsatzes unter dem Einfluß $\beta$ von Insulin, daß einerseits durch Insulin die Galaktoseabsorption im Darm gehemmt wird, andererseits das Verteilungsvolumen für Galaktose vergrößert wird.

Tab. 1

Kinetische Größen bei oraler Galaktosebelastung Lebergesunder ọnne und mit gleichzeitiger Insulingabe

\begin{tabular}{lccccccccc}
\hline & $\mathrm{K}_{\mathrm{M}}$ & $\mathrm{ZK}$ & $\mathrm{K}_{\mathrm{D}}$ & $\mathrm{ZK}$ & $\mathrm{K}_{\mathrm{I}}$ & $\mathrm{ZK}$ & $\mathrm{K}_{\mathrm{B}}$ & $\mathrm{ZK}$ & $\mathrm{A}$ \\
\hline Ohne Insulin & 0,0315 & $\mathbf{3 1 , 7}$ & $\mathbf{1 , 6 7 5 0}$ & $\mathbf{0 , 6 0}$ & $\mathbf{0 , 0 2 6 0}$ & $\mathbf{3 8 , 5}$ & $\mathbf{0 , 0 6 7 5}$ & $\mathbf{1 , 4 8}$ & $\mathbf{4 , 0}$ \\
Nach Insulin & $\mathbf{0 , 0 3 1 5}$ & $\mathbf{3 1 , 7}$ & $\mathbf{0 , 1 2 5 0}$ & $\mathbf{8 , 0 0}$ & $\mathbf{0 , 0 2 6 0}$ & $\mathbf{3 8 , 5}$ & $\mathbf{0 , 1 1 0 0}$ & $\mathbf{9 , 1}$ & $\mathbf{4 , 0}$ \\
\hline
\end{tabular}




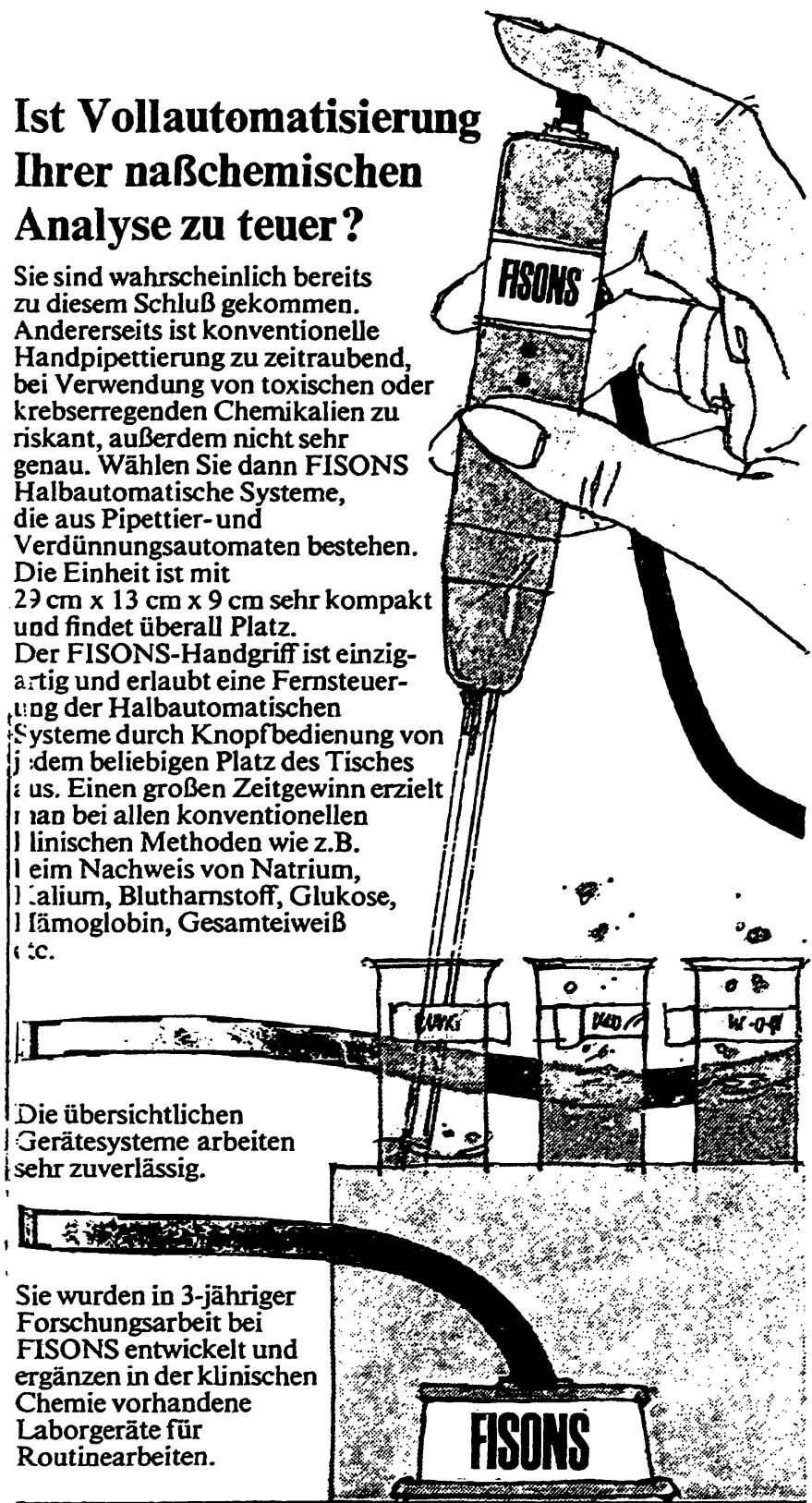

\section{A HoDis}

Fisons Limited International Division,

9 Grosvenor St, London W1X OAH
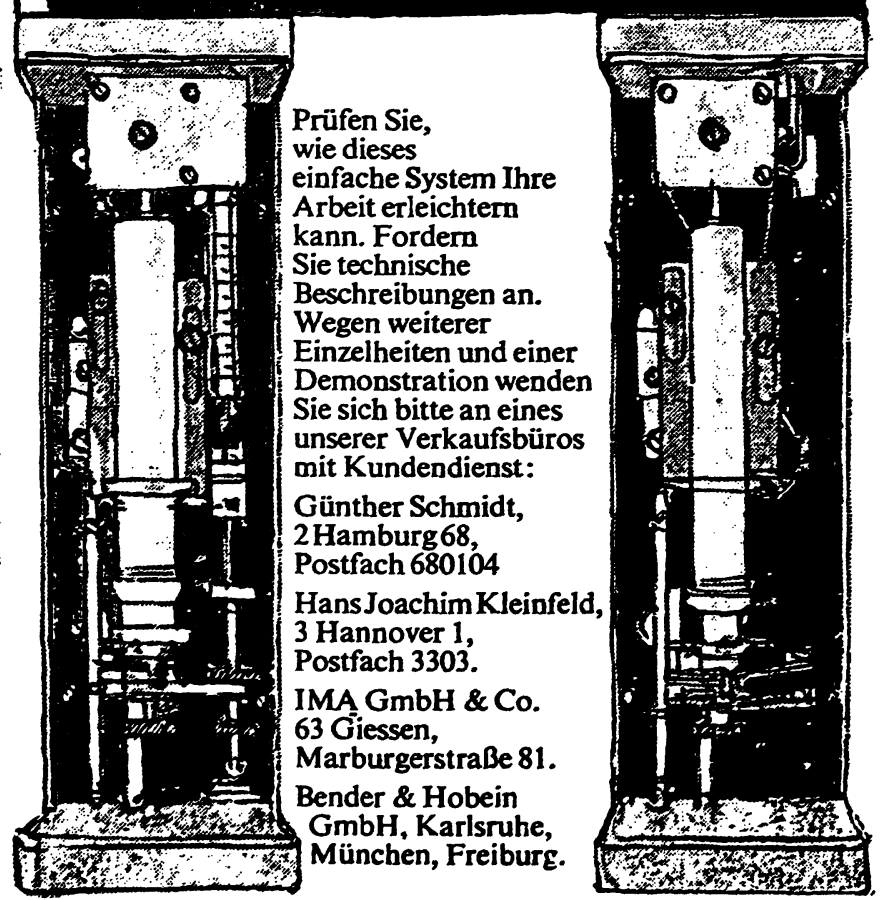

6 PSA 17

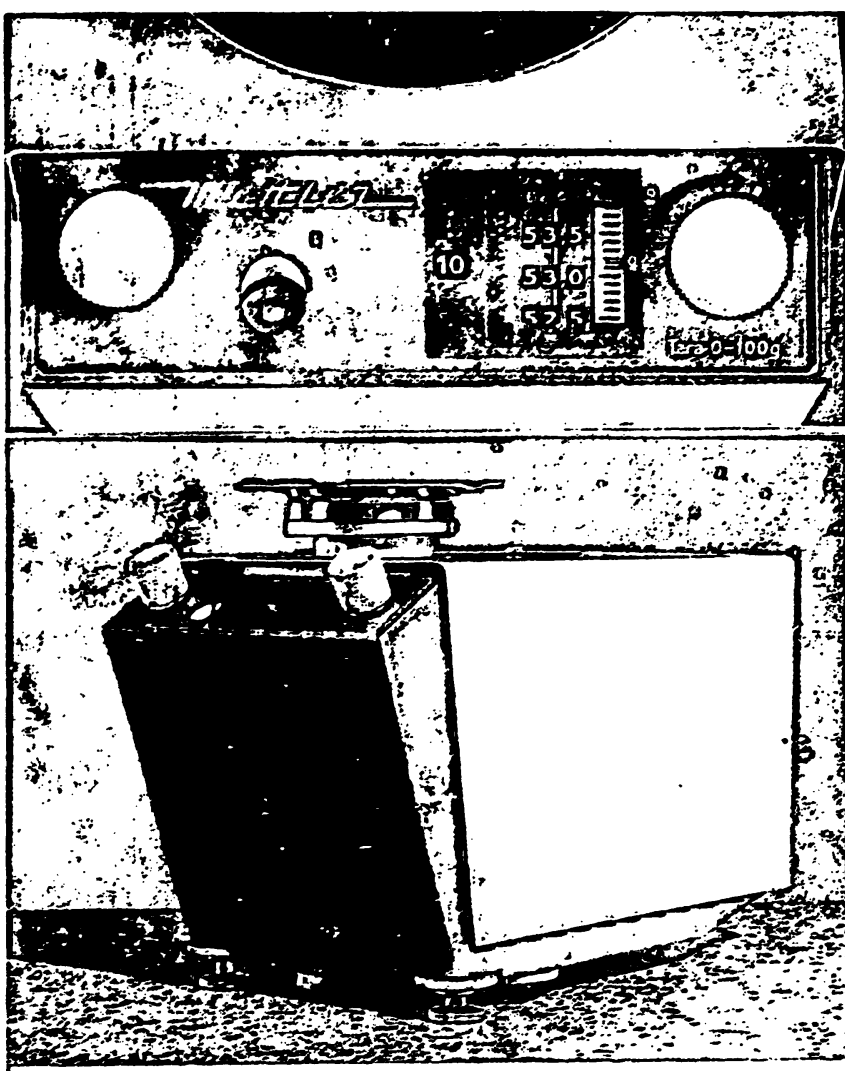

\section{Mettler-Analysen- und Präzisionswaagen}

hat unsere Gesellschaft von April 1950 bis Mai 1968 in Deutschland verkauft. Ein beispielloser Erfolg! Wir haben in den Jahren 1950 bis 1957 die Marke Mettler auf dem deutschen Markt eingeführt und fast zwei Jahrzehnte lang an der starken Verbreitung moderner Feinwaagen maßgebend mitgewirkt. Wir beraten und beliefern Sie gern.

\section{Autorisierte Mettler-Vertretung: Colora Messtechnik GmbH 7073 Lorch/Württ., Postfach 5 T (07172) 6041, FS 07-248886}

Technische Büros (Verkauf und Kundendienst): 1000 Berlin 30, KurtürstenstraBe 84, T 135200 2000 Hamburg 19, Osterstraße 63, T 400606 , FS 02-12947 3000 Hannover, An der Tiefenriede 45, T 884500 4000 Düsseldorf, Kronprinzenstr. 62, T 17860 , FS 08-587 253 6000 Frankfurt/M., Rōderbergweg 4-6, T 446031, FS 04-11 216 7073 Lorch/Württ., Barbarossastr. 3, T 6041, FS 07-248 886 8000 München 2, Dachauer Straße 175, T 5169858

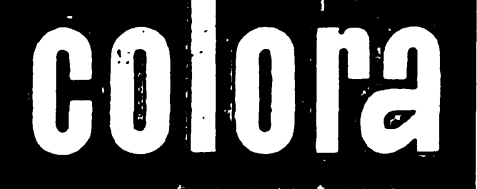




\title{
Hoppe-Seyler's Zeitschrift für Physiologische Chemie
}

\author{
Herausgegeben von: A. ButenANDt - K. Thomas - G. Wettzer \\ Redaktion: A. Dirlmanin • G. Petres
}

Bezugspreis: Ein Jahrgang zu zwölf Heften DM 360,-; Einzelheft DM 34,-

\section{Inhaltsverzeichnis von Band 350, Heft 4 / April 1969}

Uber das Syntheseprodukt der Fettsäuresynthetase aus den Lebern verschiedener Wirbeltiere

D. Eberhagen, H. Mrosek, N. Pfanzelt und W. Seitz

Uber das Sulfochinovosyl-diacylglycerin aus höheren Pflanzen, Algen und Purpurbakterien

A. RADUNZ

Untersuchungen über Zusammensetzung und Kristallstruktur von Knochensalz durch Derivatographie und Infrarot-Spektrophotometrie

F. Paulik, K. Eröss, J. Pauzik, T. Farkas and T. VizkeleIr

Uber die Hydrolyse von Carboxymethylchitin durch Lysozym

A. MARzotTo and L. GALzigna

Kristallisierte Leucinaminopeptidase aus Rinderaugenlinsen. Bestimmung der Gestalt und der Hydratation durch Röntgen-Kleinwinkelstreuung

K. Kretschmer und G. Kollin

Untersuchungen zum Einfluß der Ascorbinsäure auf die Aktivität und die Biosynthese mischfunktioneller Oxygenasen sowie auf den Gehalt an Hämoproteiden in der Mikrosomenfraktion der Meerschweinchenleber

H.-W. Leber, E. Degkwitz und Hj. Staudinger

Säulenchromatographische Abtrennung von Xanthommatin aus biologischen Extrakten. Mikromethode

H. WENZI

Glykosaminoglykan-Peptide aus dem humanen Kniegelenkknorpel

H. Greiring und H. W. StuhisatZ

V. Mitteilung über Pyrimidine. 5-Brom-uracil-4-carbaldehyd

R. Brossmer und D. ZIEGLER

n-Triacontanal und andere langkettige Aldehyde in den Oberflächenlipiden von Pflanzen

H. H. O. SCHMID and P. C. BANDI

Zur Auftrennung und Charakterisierung immunkompetenter Zellen mit Hilfe der trägerfreien Ablenkungselektrophorese

K. HANNIG und $K$. ZEILLER

Markierung essentieller Aminosäurereste der Lactat-Dehydrogenase aus Schweineherz mit [Carbonyl-14 C]-3-[2-Brom-acetyl]-pyridin

Chr. Woenckhaus, J. Berghüuser und G. Pfeeiderer

Nachweis eines Methionylrestes im aktiven Zentrum der cytoplasmatischen Malat-Dehydrogenase aus Schweineherz

V. Leskovac and G. PFLEIDERER

Erneute Untersuchung der Struktur von Acylgalaktosyl-diglycerid aus Spinatblättern

E. Heinz and A. P. Tulloch

Trennung, Isolierung und Bestimmung von Proto-, Kopro-, Pentacarboxy-, Hexacarboxy-, Heptacarboxy- und Uroporphyrin M. Doss

Uber Ricin; Reinigung und Differenzierung der Wirkungen (XVIII. Mitteilung über Samenproteine)

E. WALDSCHMTDT-LEITZ und L. KeLLER

Isolierung und Untersuchung kreuzreagierender Antikörper gegen Human- und Schweineserum- $\beta$-Lipoprotein

G. Kostner, B. GENSER und A. Holasex

Eine Reaktion zur Bestimmung von Testosteron in Extrakten von Körperflüssigkeit

$$
\text { G. ITTRICH }
$$

KURZMITTEILUNGEN

Uber eine Kohlenhydrat-Protein-Bindung in $\operatorname{der} \beta$-Fructofuranosidase

H. Greiling, P. Vögele, R. Kisters und H. D. OhLenduusci

Aktivierung von Gerinnungs- und Kininsystem durch eine Plasmaesterase (HAGEMANN-Faktor): Reinigung und Wirkungsbedingungen

H. Temme, R. Jahrreiss; E. HabermanN und F. Zithike
The synthesis products of fatty acid synthetase from the livers of different vertebrates

The sulphoquinovosyl-diacyl glycerol from higher plants, algae and purple bacteria

Investigation of the composition and crystal structure of bone salt by derivatography and infrared spectrophotometry

On the enzymatic hydrolysis of carboxymethylchitin by lysozyme Crystalline leucine aminopeptidase from bovine lens. Determination of the shape and hydration by small angle X-ray diffraction

Studies on the effect of ascorbic acid on the activity and biosynthesis of mixed function oxygenases and on the concentration of haemoproteins in the microsome fraction of guinea pig liver

Isolation of xanthommatin from biological extracts by column chromatography. A micromethod

Glycosaminoglycan-peptides from human kneejoint cartilage

Pyrimidines, V: Synthesis of 5-bromo-uracil-4-carbaldehyde

$\mathrm{n}$-Triacontanal and other long-chain aldehydes in the surface lipids of plants

Separation and characterisation of immunocompetent cells with the aid of the free flow continuous electrophoresis

Labelling of the essential amino acid residues of pig heart lactate dehydrogenase with [carbonyl-14 $\mathrm{C}] 3-(2$-bromoacetyl)pyridine

Evidence for a methionyl residue in the active site of the cytoplasmic malate dehydrogenase from pig heart

Reinvestigation of the structure of acylgalactosyldiglycerride from spinach leaves

Separation, isolation and determination of proto-, pentacarboxy-, hexacarboxy-, heptacarboxy- and uroporphyrin

Purification of ricin and differentiation of its actions (XVIIIth Communication on seed proteins)

Isolation and study of cross reacting antibodies to human and swine serum $\beta$-lipoprotein

A reaction for the determination of testosterone in extracts of body fluids

Short Communications

A carbohydrate-protein bond in $\beta$-fructofuranosidase

Activation of the coagulation system and the kinin system by an esterase from blood plasm (Hagkman factor): Purification and conditions of activity 


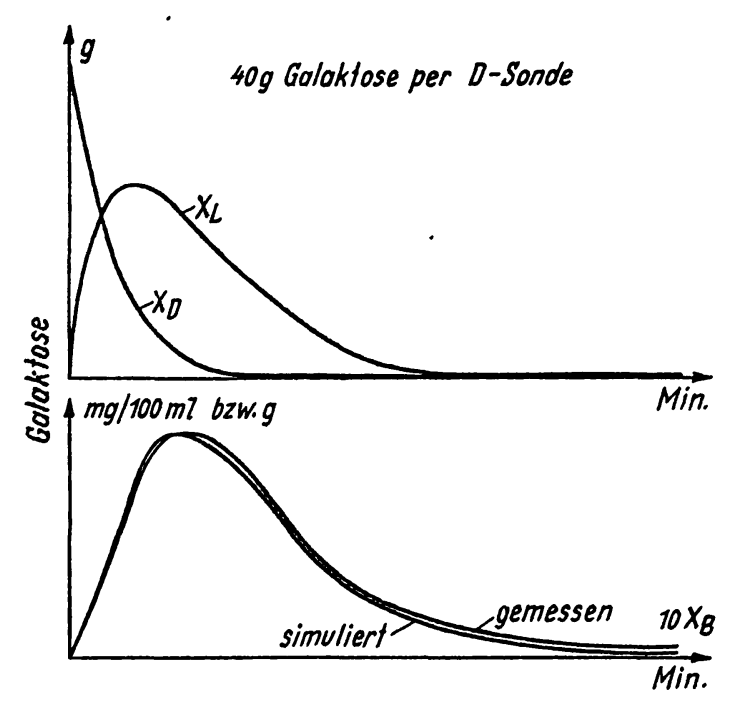

Abb. 5

Bimulation des Galaktoseumsatzes (unten) und Compartmentanalysen (oben) nach Galaktosegabe durch eine Duodenalsonde

\section{Diskussion}

der Ergebnisse über den Insulineinfluß auf den Galaktoseumsatz

Der Einfluß von Insulin auf den Galaktoseumsatz ist bisher vorwiegend an eviscerierten Tieren untersucht worden. In erster Linie befaßte sich damit die Arbeitsgruppe von Levine $(6,7,9)$. Diese Autoren konnten bei eviscerierten Tieren zeigen, daß durch Insulin der Galaktoseaustritt aus der Blutbahn beschleunigt und das Galaktoseverteilungsvolumen vergrößert wird, und zwar von $40-45 \%$ auf $70 \%$ des Körpergewichtes. Damit verteilt sich die Galaktose unter Insulineinfluß auf das Gesamtkörperwasser. Insulin bewirkte also einen Transfer der Galaktose ins Zellinnere. Zu gleichen Ergebnissen kamen auch andere Untersucher wie HUYCKE und KURFøFFER (8), die feststellten, daß Insulin die Abflußrate von Galaktose aus den Extrazellulärräumen der isoliert perfundierten Hinterpfote der Katze steigerte. Park und Johnson (19) fanden unter Insulin am eviscerierten Tier einen Anstieg von Galaktose im M. gastrocnemius, im Herzmuskel und im Diaphragma, nicht aber im Gehirn.

Durch diese tierexperimentellen Befunde ist also gesichert, daß Insulin zu einer Vergrößerung des Verteilungsraumes für Galaktose infolge eines verstãrkten Abstroms ins Zellinnere führt. Diese Ergebnisse erklären zunächst die Abflachung der Galaktoseblutspiegelkurve nach Insulingabe. Die Ergebnisse der Analogrechneranalyse stimmen damit überein, denn sie ergaben ebenfalls eine Steigerung der Galaktoseclimination aus dem Blut bzw. Kreislauf. Die Analogrechncranalyse stellt aber auch die Hypothese auf, daß Insulin die Gclaktoseabsorption im Darm hemmt. Diese Möglichkeit wird in den angeführten Untersuchungen am eviscerierten Tier nicht berücksichtigt. v. UexküLL (5) hatte den Einfluß von Insulin auf den Galaktosestoffwechsel zwar am intakten Tier nach
Verabfolgung der Galaktose mittels Schlundsonde untersucht, in seinen Bilanzbetrachtungen aber eine „Restgalaktose“ errechnet, über deren Verbleib keine Aussage gemacht werden konnte, insbesondere konnte die „Restgalaktose" nicht als Muskel- oder Leberglykogen wiedergefunden werden. Tierexperimentell wurde diese Frage systematisch und sehr gründlich von Beyreiss, Müller und Strack (4) untersucht. Diese Autoren untersuchten unter Dauerinfusion mit Galaktose in den Dünndarm den Gelaktosegehalt in der Pfortader und Vena jugularis und fanden, daß intravenös infundiertes Insulin die Galaktoscresorption um etwa $35 \%$ vermindert. Nach ihren Untcrsuchungen scheint Insulin die aktive Galaktoseresorption wcitgehend auszuschalten. Die Hemmung der Galaktoseresorption durch Insulin war nicht durch cine Hypoglykämie bedingt. Die direkte Messung des Galaktosegehaltes des durchgespülten Darms ergab unter Insulin eine etwa vicrmal so große Menge nicht resorbierter Galaktose wie ohne Insulingabe. Beykerss und Mitarbeitet (4) fanden im übrigen ebenfalls unter Insulingabe eine Vergrößerung des Verteilungsraumes der Galaktose.

Diese tierexperimentell erhobenen Befunde bestãtigen in eindrucksvoller Weise die am Analogrechner aufgestellte Hypothese, daß Insulin die Resorption der Galaktose hemmt.

Zusammenfassend können wir feststellen, daß die Erniedrigung der Galaktoseblutspiegelkurve durch Insulin eine Folge von zwei Vorgängen ist. Erstens wird das Verteilungsvolumen für Galaktose und damit der Transport aus der Blutbahn ins Zellinnere beschleunigt, als Folge davon kommt es zu einer Abflachung des Blutspiegels und zweitens wird die Resorption der Galaktose im Darm gehemmt. Unter diesem Aspekt lassen die Resorptionsuntersuchungen von HoLdsworth und Dawson (14) eine neue Hypothese zu. Dicse Autoren fanden nämlich bei Darmperfusionsuntersuchungen am Menschen eine Hemmung der Galaktoseresorption nach Zugabe von Glucose. $\mathrm{Da}$ die Insulinaktivität bei diesen Untersuchungen nicht gemessen wurde, scheint es nicht ausgeschlossen, daß die Hemmung der Galaktoseresorption von einer Insulinausschüttung mit beeinflußt wird. Über die vorliegende Untersuchung hinaus soll die Analogrechneranalyse des Galaktosestoffwechsels anregen, Computeranalysen in die experimentelle und klinische Medizin einzuführen. Dieses Verfahren, dessen mathematische und rechentechnische Lösung als Grundmodell für die meisten Stoffwechsclvorgänge nach oraler Stoffverabreichung gelten darf, dürfte damit der Verdauungs-, Resorptionsund Stoffwechselforschung neue Möglichkeiten eröffnen. Ein prinzipielles Analogrechnermodell für die Stoffwechselkinetik intravenös zugeführter Stoffe wurde von AdAM, Schröder, RAptis und Pfeipfer (20) vorgestellt. Für dic klinische Laboratoriumsmedizin entsteht damit eine neue Möglichkeit, zur Untersuchung der Dynamik des Stoffwechsels und Organleistungen Gesunder und Kranker. 


\section{Literatur}

1. Pollak, L. und G. Fehér, Naunyn-Schmiedebergs Arch. exper. Path. 172, 407 (1933). - 2. Shay, H., E. M. Schross und M. A. BeLL, The metabolism of galactose. I. Consideratione underlyng the use of galactose in tests of the function of the liver. Arch. Int. Med. Chicago 47, 391 (1931). - 3. WierzuKkowski, M., Biochem. Z. 237, 92 (1931). - 4. BeyreIss, K., F. Müller und E. Strack, Zschr. exper. Med. 138, 277 (1964). 5. v. UexKüLL, TH., Zschr. klin. Med. 140, 327 (1942). - 6. Goldstein, M. S., Mendel und R. Levine, Amer. J. Physiol. 163, 714 (1950). - 7. Goldstein, M. S., W. L. HenRY, B. Huddiestun und R. Levine, Amer. J. Physiol. 173, 207 (1953). - 8. HuYCKe, E. J. und P. KRuhøFFER, Acta physiol. Scand. 34, 232 (1955). 9. Levine, R., M. S. Goldsten, B. Huddlestun und S. P. Klein, Amer. J. Physiol. 163, 70 (1950). - 10. Wick, A. N. und D. R. DruRy, Amer. J. Physiol. 173, 229 (1953). - 11. Rommer, K., F. Melani, K. Grimmel und H. Burkhardt, Diabetologica (im
Druck). - 12. Hunt, J. N. und W. R. SPURReLL, in: Bockus, H. L.: Gastroenterology. Volume 1, S. 286, W. B. Saunders Comp. Philadelphia (1966). - 13. Wrrson, T. H., Intestinal Absorption. Philadelphia (1962). - 14. Holdsworth, C. D. und A. M. DAwson, The absorption of monosaccharides in men. Clin. Sc. London 27, 371 (1964). - 15. LINNEWEH, F., E. SCHAUMLÖPFEL und W. BARTHELMat, Klin. Wischr. 43, 405 (1965). - 16. Moseley, V. und F. W. CHORNOCK, J. clin. Inveșt. 26, 11 (1947). - 17. WALDstein, S. S., L. A. Greenburg, A. D. Biggs und L. Corn, J. Laborat. clin. Med. S. Louis 55, 462 (1960). - 18. Rommer, K. E. Bernt, F. Schmitz und K. Grimmer, Klin. Wsschr. 46, 936 (1968). - 19. Park, C. R. und L. H. Johnson, Amer. J. Physiol. 182, 17 (1955). - 20. Adam, E. W., S. Raptis, K. E. Schröder und E. F. Pfeiffer, 8. Internat. Symposion Bad Gastein vom 9.-12.1.68 (im Druck).

Priv.-Doz. Dr. K. Rommel $7900 \mathrm{UIm} /$ Donau Steinhövelstr. 9 